\title{
Structural Analysis of Clostridium difficile Toxins A and B Using a Hybrid Approach
}

\author{
R. N. Pruitt,* M. G. Chambers, ${ }^{* *}$ D. B. Lacy, ${ }^{*}$ and M. D. Ohi**
}

*Departments of Microbiology and Immunology, and Biochemistry, Vanderbilt University School of Medicine, Nashville, TN 37232

** Department of Cell and Developmental Biology, Vanderbilt University School of Medicine, Nashville, TN 37232

C. difficile is a gram-positive, spore-forming anaerobe that infects the colon, causing a range of disorders including diarrhea, pseudomembranous colitis, and toxic megacolon $(1,2)$. While $C$. difficile does not generally infect healthy individuals, it poses a threat to individuals with a depleted natural intestinal flora. Thus, patients undergoing antibiotic treatment are most at risk of acquiring $C$. difficile associated disease (CDAD). C. difficile is encountered most often in nursing home and hospital settings, where there are large populations of infected and at risk individuals. Kyne et al. estimated that in 1999, the financial burden of CDAD on the US healthcare system was $\$ 1.1$ billion per year (4). Since that time, there has been a dramatic increase in both the prevalence and the severity of $C$. difficile infections due to the emergence of the hypervirulent strain NAP1/027 $(5,6)$. The emergence of this new strain underscores the significance of $C$. difficile as one of the most threatening nosocomial pathogens.

Clostridium difficile toxins $\mathrm{A}$ and $\mathrm{B}$ are the main cause of pseudomembranous colitis and hospital-acquired diarrhea. Both members of a family of large clostridial toxins (LCT), the structure and mechanism of these proteins have been poorly characterized. TcdA and TcdB are central targets in the prevention and treatment of $C$. difficile-associated disease. As such, it is essential that we gain a structural understanding of how the toxins are organized and their structures change to gain entry into host cells. TcdA and B contain four functional domains that are associated with host-receptor binding, membrane pore formation, autoprocessing, and the enzymatic modification of host Rhofamily proteins.

TcdA and TcdB were purified from C. difficile culture supernatants and shown to be active in a cell-rounding assay. Toxin was adsorbed to carbon-coated glow-discharged grids, stained with uranyl formate. TcdB particles were clearly more heterogeneous than the TcdA toxin; however, analysis of $\sim 2,500 \mathrm{TcdB}$ particles and $\sim 3,000 \mathrm{TcdA}$ particles into class averages show that these two toxins share a similar structural organization. A 3D reconstruction of TcdA was calculated to $\sim 24 \AA$ resolution using the random conical tilt approach (Fig. 1B). The molecule has a 'pincher-like' head and two tails, long and short. Expression of recombinant fragments indicates that the 'pincher-like' head corresponds to the delivery domain while the long tail corresponds to the receptor-binding domain. Inducing the auto-proteolytic processing event resulted in removal of the short tail, revealing that this domain corresponds to the glucosyltransferase domain. The location of the autoprotease domain within the map of TcdA was not experimentally determined, but the structure is anticipated to be located so that the C-terminus of the glucosyltransferase domain can be cleaved in the active site of the protease. The TcdB glucosyltransferase and TcdA autoprocessing domain X-ray crystal structures along with a model of the TcdA receptor-binding domain were manually docked into the experimental EM density (Fig. 1A).

To understand the structural basis for pore formation within the endosome, we analyzed TcdA particles at low $\mathrm{pH}$. TcdA was applied to a carbon-coated, glow-discharged grid, washed with 
a $\mathrm{pH} 4.5$ buffer, and then stained with uranyl formate. The 3D structure of TcdA at low pH shows a molecule that has undergone a major conformational change from the structure observed at neutral $\mathrm{pH}$ (Fig. 1C). Although the conformation of the binding domain has not changed, the low $\mathrm{pH}$ form is more elongated due to an extension of the head domain. The low $\mathrm{pH}$ structure of TcdA has a volume that corresponds to a MW of $\sim 240 \mathrm{kDa}$ and is thus considerably smaller than the structure of native TcdA whose volume corresponds to a MW of $\sim 320 \mathrm{kDa}$. This difference may be due to greater flexibility and/or partial unfolding of the glucosyltransferase domain at low $\mathrm{pH}$.

\section{References}

[1] D.E. Voth and J.D. Ballard. Clin Microbiol Rev 18 (2005) 247-263.

[2] D.M. Lyerly, H.C. Krivan, and T.D. Wilkins, Clin Microbiol Rev 1, (1988) 1-18.

[3] D.M. Lyerly, et al. Infect Immun 47 (1985) 349-352.

[4] L. Kyne, et al. Clin Infect Dis 34 (2002) 346-353.

[5] C.P. Kelly, and J.T. LaMont. N Engl J Med 359 (2008) 1932-1940.

[6] J. Cloud, and C.P. Kelly. Curr Opin Gastroenterol 23 (2007) 4-9.

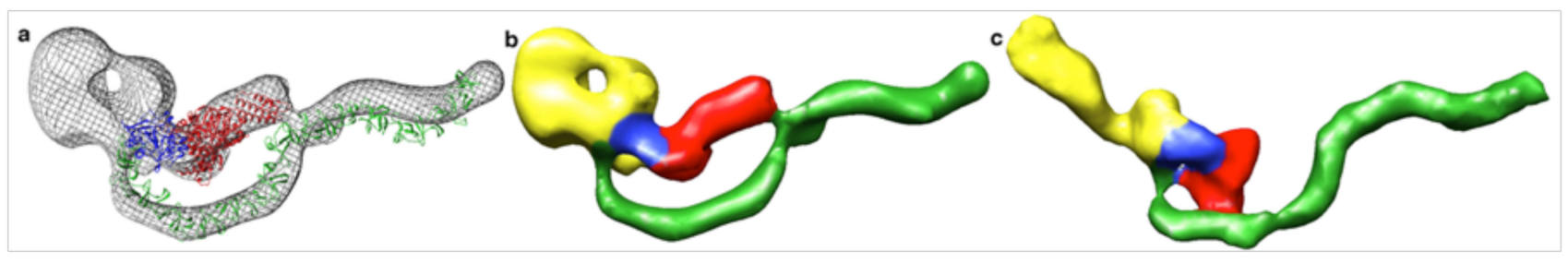

FIG. 1. The 3D structure of TcdA has been obtained at neutral and low $\mathrm{pH}$ by electron microscopy. a) The structural models for the glucosyltransferase (red), autoprotease (blue), and receptor-binding (green) domains have been docked into the neutral $\mathrm{pH}$ density, but at present, no structural models for the delivery domain exist. b) The structure at neutral $\mathrm{pH}$ reveals a pincher-like head domain (yellow) and two tails, short and long. Colored as in Fig. 1a. c) The structure at the low $\mathrm{pH}$ of the endosome reveals significant change in the TcdA head region 